\title{
Optimal Feeding Strategy for Bioreactors with Biomass Death
}

\author{
L. Bodizs, B. Srinivasan, D. Bonvin \\ Laboratoire d'Automatique, \\ Ecole Polytechnique Féderale de Lausanne, \\ CH-1015, Lausanne, Switzerland
}

\begin{abstract}
Optimization of bioreactors, and especially the maximization of product yield, has been studied extensively in the literature. It has been shown that, in many cases, the optimal solution corresponds to keeping the substrate concentration constant at a value that maximizes the instantaneous yield. However, in the presence of biomass death, keeping the substrate concentration constant at this value may lead to biomass extinction, i.e. no active biomass left in the reactor. In such a case, the optimal solution arises from a compromise between avoiding extinction and increasing the instantaneous yield. In addition, if the maintenance term depends on the amount of inactive biomass, the optimal solution requires a time-varying substrate concentration. These issues are illustrated via the optimization of the batch filamentous fungi fermentation.
\end{abstract}

\section{Introduction}

For centuries, man has employed biological processes to his benefit for the production of bread, cheese, wine, beer, and yoghurt. Due to the importance of these products, scientists have studied the way these reactions take place inside the microorganisms and have exploited their capabilities to perform more complex and more useful transformations (Bailey \& Ollis 86). The other major development in biotechnology is the use of mathematical modeling and subsequent application of control and optimization techniques in order to improve the performance of bioprocesses. Numerous models of varying complexity have been proposed, i.e. from simple tendency models to detailed ones that use partial differential equations (Bailey \& Ollis 86, Lee 92).

The optimal feeding strategy in bioreactors depends on the structure of the reaction kinetics and the interaction between the different reactions. Using a fairly general structure, many useful results have been derived in (Modak et al. 86, van Impe et al. 94). One key result is that, in many cases, the final product yield (ratio of the amount of product formed and the amount of substrate consumed) can be maximized simply by maximizing the same instantaneously. However, this is not necessarily true with biomass death. In the context of operational optimization, the death of biomass has rarely been discussed in the literature. The reason for this negligence is the fact that biomass death does not fundamentally affect the structure of the solution, unless extinction of biomass becomes important. Extinction is the phenomenon where the rate of death is larger than the rate of growth and no more active biomass is left before the end of the batch.

In this paper, a scenario is considered where maximizing the product yield instantaneously corresponds to extinction of the biomass. The type of kinetics and the interplay between the parameters that can lead to such a scenario are discussed. It is shown that, with biomass death, the optimum still corresponds to a constant substrate concentration, but the value is shifted such that extinction occurs just at the end of the batch. Also, when the inactive biomass has to be maintained, the optimal substrate concentration is time varying. 
The basic concepts will first be presented using a general model, and later the same will be illustrated in simulation via the optimization of a batch filamentous fungi fermentation. Here, the tendency model developed by (Agger et al. 98) will be used. The results show that, though a more complex system of equations is used, the conclusions drawn from a simple structural model are indeed valid.

The paper is organized as follows. In the next section, the key aspects of the optimization problem are discussed using a model with unspecified kinetics. The role of biomass death with regard to optimization will be dealt with in Section 3. Simulation results from the filamentous fungi fermentation are presented in Section 4, and conclusions drawn in Section 5 .

\section{Optimization of Fed-batch Bioreactors}

\subsection{A General Model with Unspecified Kinetics}

The optimization of fed-batch processes with a general model with unspecified kinetics of the type given below is widely discussed in the literature (Modak et al. 86, van Impe et al. 94):

$$
\begin{aligned}
& \frac{d m_{x}}{d t}=\square(s) m_{x} \\
& \frac{d m_{s}}{d t}=\square(s) m_{x}+s_{f} F \\
& \frac{d m_{p}}{d t}=\square(s) m_{x} \\
& \frac{d V}{d t}=F
\end{aligned}
$$

where $V$ is the volume, $m_{s}$ the mass of substrate, $s=m_{s} / V$ the concentration of substrate, $m_{x}$ the mass of biomass, $x=m_{x} / V$ the concentration of biomass, $m_{p}$ the mass of product, $p=m_{p} / V$ the concentration of product, $s_{f}$ the inlet substrate concentration, $F$ the feed flow rate, $\square(s)$ the specific biomass growth rate, $\square(s)$ the specific substrate consumption rate, and $\square(s)$ the specific production rate.

The development in this paper relies on certain key concepts that are explained next:

- Maintenance: This corresponds to substrate being consumed to sustain the existing biomass. Mathematically, it is represented as an additional term in the substrate consumption rate $\square$. Note that, with this definition, the amount of biomass does not decrease even when no substrate is present.

- Death of biomass: This corresponds to transforming the (active) biomass into a nonproducing (inactive) form. In this case, it is important to distinguish between the two forms (active and inactive) since only the active one is useful for product formation. Inactivation is represented mathematically as a negative term in the specific growth rate $\square$. Thus, if the substrate concentration $s$ is close to zero, the amount of active biomass will decrease since the negative term becomes dominant.

- Extinction: If the specific growth rate $\square$ is negative, then the quantity of active biomass reduces and eventually goes (asymptotically) to zero. Note that the amount of dead biomass does not go to zero but converges to a large constant value. This phenomenon is similar to washout in continuous reactors, which occurs at high dilution rates. 
- Extinction limit $s_{\text {ext }}$ : This is the value of $s$ below which $\square<0$ and extinction occurs.

\subsection{Optimization Problem Formulation and Solution}

Consider the terminal-cost optimization problem:

$$
\begin{aligned}
& \max _{t_{f}, F(t)} J=\frac{m_{p}\left(t_{f}\right)}{m_{s}(0) \square m_{s}\left(t_{f}\right)+s_{f}\left(V\left(t_{f}\right) \square V(0)\right)} \\
& \text { s.t. dynamic model (1) } \\
& F_{\text {min }} \square F(t) \square F_{\text {max }}
\end{aligned}
$$

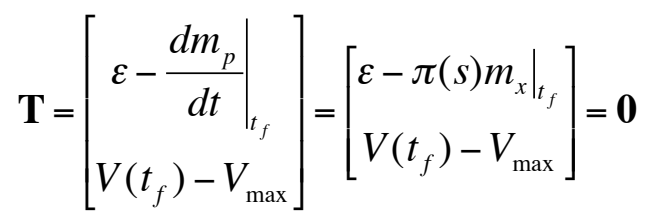

where $J$ is the scalar performance index and $\mathbf{T}$ the vector of terminal equality constraints. The performance index corresponds to maximizing the final product yield, i.e. the mass of product formed per g. of substrate consumed. Ideally, the process would stop when $d m_{p} / d t$ reaches zero. However, this happens only for $t \square$ because of the exponential decay of $m_{p}$. Consequently, to have a finite terminal time, $d m_{p} / d t \geq \square>0$ is chosen as a terminal constraint. The second terminal constraint is related to the desired final volume.

It was shown in (Modak et al. 86, van Impe et al. 94) that the optimal feed rate profile consists of three types of intervals: (i) maximum feed rate $F(t)=F_{\max }$, (ii) minimum feed rate $F(t)=0$, and (iii) sensitivity-seeking $\operatorname{arc} F(t)=F_{\text {sens }}(t)$ determined by intrinsic compromises present in the system.

The derivation of the analytical expression for $F_{\text {sens }}(t)$ is based on Pontryagin's minimum principle and is detailed in (Modak et al. 86). However, some of the reasoning is reproduced here briefly. Model (1) can be written as:

$$
\dot{\mathbf{x}}=\mathbf{f}(\mathbf{x})+\mathbf{g} F
$$

where

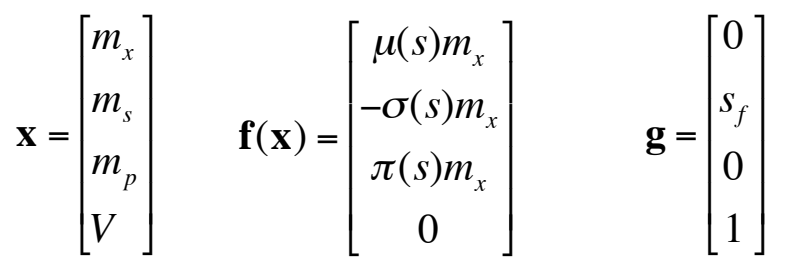

The Hamiltonian in this case is:

$$
H=\square[\mathbf{f}(\mathbf{x})+\mathbf{g} F]=\square \mathbf{f}(\mathbf{x})+\square \mathbf{g} F=\square(\mathbf{x}, \square)+\square(\square) F
$$

where $\mathrm{Q}$, the vector of adjoint variables, satisfies the following dynamics:

$$
\dot{\square}^{T}=\square \frac{\partial H}{\partial \mathbf{x}}=\square \square^{T} \frac{\partial \mathbf{f}(\mathbf{x})}{\partial \mathbf{x}}, \quad \square^{T}\left(t_{f}\right)=\left.\frac{\partial J}{\partial \mathbf{x}}\right|_{t_{f}}+\square^{T}=\left.\frac{\partial \mathbf{T}}{\partial \mathbf{x}}\right|_{t_{f}}
$$

The expressions for $\square$, the switching function, and $\square$ are given in (6) and (7): 


$$
\begin{aligned}
& \square(\square)=\square_{2} s_{f}+\square_{4} \\
& \square(\mathbf{x}, \square)=\left(\square_{1} \square \square \square_{2} \square+\square_{3} \square\right) m_{x}
\end{aligned}
$$

The adjoint equations (5) become:

$$
\begin{aligned}
& \dot{\square}_{1}=\square\left(\square_{1} \square \square \square_{2} \square+\square_{3} \square\right) \\
& \dot{\square}_{2}=\square\left(\square_{1} \square \square \square \square_{2} \square \square+\square_{3} \square \square m_{x} / V\right. \\
& \dot{\square}_{3}=0 \\
& \dot{\square}_{4}=\square\left(\square_{1} \square \square \square_{2} \square \square+\square_{3} \square \square m_{x} m_{s} / V^{2}\right.
\end{aligned}
$$

where $\square \square=(\partial \square / \partial s), \square \square=(\partial \square / \partial s)$, and $\square \square=(\partial \square / \partial s)$. The necessary condition of optimality give:

$$
F=\begin{aligned}
& F_{\text {max }} \text { for } \square<0 \\
& F_{\text {min }} \text { for } \square>0 \\
& F_{\text {sens }} \text { for } \square=0
\end{aligned}
$$

Along the singular arc $F_{\text {sens }}$, the switching function $\square$ and its time derivatives are zero. This information is used to compute $F_{\text {sens }}: \dot{\Pi}=0$ implies that $d\left(\square_{2} s_{f}+\square_{4}\right) / d t=0$, i.e. $\square_{1} \square \square \square \square \square \square$ $+\square_{3} \square \square=0$. This, with (9)-(11), implies that $\square_{2}, \square_{3}$ and $\square_{4}$ are constant over a singular interval. As described in (Bryson \& Ho 75, Srinivasan et al. 03), the input along the singular arc can be computed from $\ddot{\Pi}=0$ and using $\square_{1}=\left(\square_{2} \square \square-\square_{3} \square \square\right] / \square[$ as:

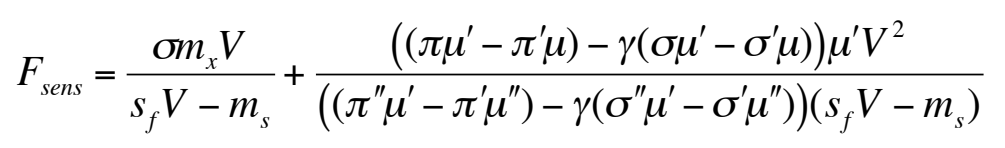

where $\square=\square_{2} / \square_{3}$ is a free parameter to be chosen.

It was shown in (Modak et al. 86) that, for a free-time problem where the performance index does not depend explicitly on the terminal time, $H^{*}=H\left(t_{f}\right)=0$. This condition determines the value of $\square=(\square \square \square-\square(\mathbb{W}) /(\square \square \square \square \square(\mathbb{d})$, which leads to:

$$
F_{\text {sens }}=\frac{\square m_{x} V}{s_{f} V \square m_{s}}
$$

In fact, $F_{\text {sens }}$ this corresponds to $d s / d t=0$, i.e. keeping the substrate concentration at some constant value $s^{*}$. Note that, when $H^{*}=H\left(t_{f}\right)=0, \square_{l}$ is also constant, leading to a constant adjoint vector throughout the singular interval.

\subsection{Final Product Yield vs Instantaneous Product Yield}

It is interesting to investigate the meaning of the optimal substrate concentration $s^{*}$. Eliminating $\square_{2}$ from $\dot{\square}_{1}=O$ and $\dot{\square}_{2}=0$ gives:

$$
\frac{\square \square \square \square \square}{\square \square \square \square \square}=\frac{d(\square / \square) / d s}{d(\square / \square) / d s}=\square \frac{\square_{1}}{\square_{3}}
$$

With the performance index and terminal constraints given in (2), $\square_{1}\left(t_{f}\right)$ and $\square_{3}\left(t_{f}\right)$ can be computed from (5) as: 


$$
\begin{aligned}
& \square_{1}\left(t_{f}\right)=\square \square_{1} \square=\square \frac{\square_{1} \square}{m_{x}\left(t_{f}\right)} \\
& \square_{3}\left(t_{f}\right)=1
\end{aligned}
$$

Assuming that $m_{x}\left(t_{f}\right) \neq 0$ results in $\square_{I} \approx 0$, because $\square$ is a very small value. Thus, (15) corresponds to choosing a substrate concentration such that $d(\square \square) / d s=0$, i.e. maximizing $(\square \square)$ at every time instant. Note that $(\square \square)$ is the ratio between the rates of product formation and substrate consumption, i.e. the instantaneous product yield. In other words, this means that, if $\square_{l}=0$, maximizing the yield at the end of the batch, $m_{p}\left(t_{f}\right) /\left(m_{s}(0)-m_{s}\left(t_{f}\right)+s_{f}\left(V_{f}-V_{0}\right)\right)$, in fact corresponds to maximizing the instantaneous yield $([\square])$.

Such a situation is highly desirable since it is possible to work with a local rather than a global objective function. Thus instantaneous optimization automatically corresponds to the optimal solution for the entire interval. This case is widely discussed in the literature.

Remark 1: Note that the maximum of ( $\square \square)$ does not correspond to the maximum of $\square$. In other words, maximizing the instantaneous product yield and the specific production rate are not equivalent. For example, if $\square$ is of Monod type, depending on the parameter values, it can so happen that $\square$ is maximized at $s=\infty$ while $(\square / \square)$ is maximized at $s=0$ ! Also, if $\square$ is of Haldane type, the maximum of the instantaneous yield ( $\square \square)$ does not correspond to the peak of the Haldane function.

\section{Compromise between Instantaneous Product Yield and Avoiding Biomass Extinction}

A practical scenario is illustrated where instantaneous product yield maximization does not maximize the final product yield. Let $s_{\text {inst }}$ be the value of the substrate that maximizes $(\square / \square)$ and $s_{\text {ext }}$ be the extinction limit. In Figure 1, the grey region corresponds to the region where extinction occurs given infinite time, i.e. $s<s_{\text {ext }}$.

\subsection{Effect of Biomass Death}

The two different cases of interest are:

- $s_{\text {inst }} \geq s_{\text {ext }}$ : (Curve I) - Here, death does not influence the optimal solution of Problem (2), which corresponds to maximizing the instantaneous product yield.

- $s_{\text {inst }}<s_{\text {ext }}$ : (Curve II) - A low value of $s$ needs to be used for improving the instantaneous yield. On the other hand, if such a low $s$ is used, there will be biomass extinction. So, a compromise between yield maximization and avoiding extinction naturally arises. The optimal substrate concentration is an intermediate value between $s_{\text {inst }}$ and $s_{\text {ext }}$. The exact value depends on the volume of the reactor. The larger the volume, the closer the optimal solution is to $s_{\text {ext }}$.

In the case of no biomass extinction (Curve I) $m_{x}\left(t_{f}\right)$ will be non-zero and $\square_{l}$ goes to zero as $\square$ goes to zero (16). Thus, as discussed in Section 2.3, the optimum corresponds to maximizing $\square \square$, i.e. keeping $s$ at $s_{\text {inst }}$.

In the case of biomass extinction (Curve II), instantaneous yield maximization, which requires $\square_{l}=0$, cannot occur since $m_{x}\left(t_{f}\right)=0$ and thus $\square_{l}\left(t_{f}\right)=\infty$ (16) thereby leading to a contradiction. So, the optimal solution will correspond to a non-zero, yet small, $m_{x}\left(t_{f}\right)$ and thus a large $\square_{l}$. The exact value of $\square_{l}$ depends on how big the reactor is. In other words, the optimal solution corresponds to maintaining the active biomass such that its concentration reaches zero only at the end of the operation. Also note that, even in the case of Curve II, the 
optimal solution corresponds to a constant substrate concentration. This arises from the fact that $H^{*}=0$, thus (14) holds, leading to $d s / d t=0$.

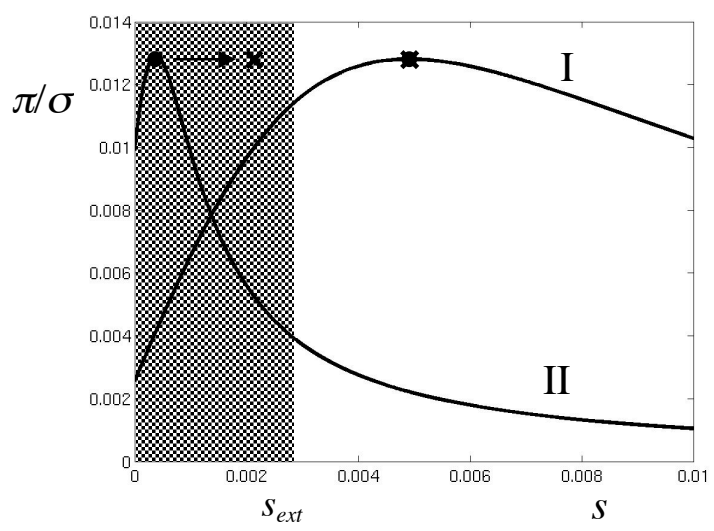

Figure 1. Interplay between avoiding extinction and instantaneous yield maximization.

$(\bullet)$ is the instantaneous yield optimum, i.e. $s_{i n s},(\mathbf{x})$ is the optimum solution.

\subsection{Maintenance of the Inactive Biomass}

So far, the terminologies of dead biomass and inactive biomass have been used interchangeably. However, in many cases when the biomass becomes inactive, it does not necessarily cease to consume some substrate for maintenance (i.e. the nomenclature of inactive is more appropriate). So, maintenance of inactive biomass becomes a burden that needs to be handled throughout the batch.

Such a situation does not directly fall in the class of problems described by Model (1), since the evolution of $m_{s}$ should include a term that depends on the inactive biomass. Thus, the results obtained from Problem (2) are no longer valid. A quick fix is to come up with an approximation of how much the maintenance would cost in terms of product yield, as described next:

- Assume that the inactive biomass increases linearly with time.

- If the maintenance is proportional to the inactive biomass, the total amount of substrate used for maintenance is proportional to the square of the batch time.

Hence, a term proportional to the square of the batch time is added to the cost function.

$$
J=\frac{m_{p}\left(t_{f}\right)}{m_{s}(0) \square m_{s}\left(t_{f}\right)+s_{f}\left(V\left(t_{f}\right) \square V(0)\right)} \square K t_{f}{ }^{2}
$$

This penalizes long batches since the maintenance would be inhibitive. The mathematical contribution of the extra term in the cost function is that $H^{*}=K t_{f} \neq 0$. So, the optimal solution no longer corresponds to a constant substrate concentration, but to a time-varying one. Yet, (13) can be used to compute the optimal input with an appropriate choice of $\square \mathrm{A}$ larger substrate concentration is used initially to grow the biomass in the first phase in order to shorten the batch. Then, the substrate concentration $s$ is reduced so as to improve the instantaneous yield as, by that time, extinction is not crucial any more. Thus, the typical feed profile will be bell-shaped. 


\section{Optimization of the Filamentous Fungi Fermentation}

In this section, the morphologically structured model of the filamentous fungi fermentation process $(\square$-amylaze production from glucose monohydrate by Aspergillus oryzae in fed-batch operation) adapted from (Agger et al. 98) is presented. Then, the results of numerical optimization will be analyzed in the light of the discussion in Section 3.

\subsection{Reaction system}

The basic characteristic of filamentous organisms is that the fungal hyphae can be split into three different regions: an extension zone representing the tips of the hyphae, an active region responsible for the growth and product formation, and an inactive hyphal region. During the evolution of the system, the active zone can transform into extension zone (branching) and it can also become inactive (death).

Accordingly, the morphologically structured model contains three types of biomass: $X_{e}$ extension zone, $X_{a}$ - active zone and $X_{h}$ - inactive zone. The reaction scheme representing the functions of the different biomass fractions and the transformation reactions can be illustrated as follows:

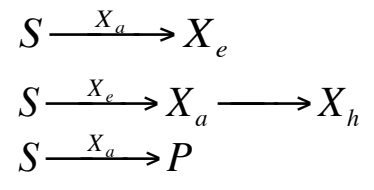

where $S$ stands for the substrate (glucose) and $P$ for the product ( $\square$-amylaze).

\subsection{Model Equations}

\subsubsection{Differential equations}

Component and total mass balances give the following model (the various variables and symbols are defined in the notation section at the end of the paper):

$$
\begin{aligned}
& \frac{d x_{e}}{d t}=q_{b} \square \frac{F}{V} x_{e} \\
& \frac{d x_{a}}{d t}=q_{a} \square q_{b} \square q_{d} \square \frac{F}{V} x_{a} \\
& \frac{d x_{h}}{d t}=q_{d} \square \frac{F}{V} x_{h} \\
& \frac{d s}{d t}=\square 1 \frac{\square}{\square} q_{a}+\frac{1}{Y_{S P}} r_{p s} x_{a}+n_{s}\left(x_{e}+x_{a}+x_{h}\right) \square+\frac{F}{V}\left(s_{f} \square s\right) \\
& \frac{d p}{d t}=r_{p s} x_{a} \square \frac{F}{V} p \\
& \frac{d V}{d t}=F
\end{aligned}
$$

\subsubsection{Algebraic equations}

The kinetic expressions read:

$$
\begin{aligned}
& r_{p s}=\frac{k_{p_{1}} s}{\left(s+K_{s 4}\right)\left(1+e^{k_{p 2}\left(s \square s_{r e p}\right)}\right)}+k_{c} \frac{s}{s+K_{c o r}} \\
& q_{b}=\frac{k_{1} s}{a\left(s+K_{s 1}\right)} x_{a}
\end{aligned}
$$




$$
\begin{aligned}
& q_{a}=\frac{k_{3} s}{s+K_{s 3}} a x_{e} \\
& q_{d}=k_{2} x_{a} \\
& k_{1}=\frac{k_{\text {bran }} \cdot 10^{4}}{\frac{\square}{4}\left(d \cdot 10^{\square 4}\right)^{2}(1 \square w) f \square} \\
& a=\frac{\square}{2} \frac{1}{2} d \cdot 10^{\square 4}-\frac{4 \square}{3}(1 \square w) \square \text { 目 } \\
& k_{3}=k_{\text {tip, max }} \cdot 10^{\square 4} \frac{\square}{4}\left(d \cdot 10^{\square 4}\right)^{2}(1 \square w) f \square \\
& d=11.25 \square+1.1 \\
& \square=\frac{q_{a}}{x_{e}+x_{a}+x_{h}}
\end{aligned}
$$

It can be observed that equations (31)-(34) form an algebraic loop: $d$ depends on $\square$ (33), $\square$ through $q_{a}$ (34) and (28) depends on $a$ and $k_{3}$, and $a$ and $k_{3}$ (31) and (32) depends on $d$. This was resolved analytically giving rise to:

$$
d=\frac{1.1+\sqrt{1.21+\frac{135 k_{\text {tip } \max } f s x_{e}}{\left(s+K_{s 3}\right)\left(x_{e}+x_{a}+x_{h}\right)}}}{2}
$$

As a result, (33) and (34) are replaced by (35).

The parameter values and initial conditions are given in Tables 1 and 2, respectively.

\begin{tabular}{|l|l|l|}
\hline Parameter & \multicolumn{1}{|c|}{ Value } & \multicolumn{1}{|c|}{ Measurement unit } \\
\hline$\square$ & 0.57 & \\
\hline$F$ & 80 & $\%$ \\
\hline$K_{2}$ & 0.08 & $\mathrm{~h}^{-1}$ \\
\hline$k_{\text {bran }}$ & 0.0017 & tip/( $\square \mathrm{m} \mathrm{h})$ \\
\hline$k_{c}$ & 8 & $\mathrm{FAU} /(\mathrm{g}$ active DW $\mathrm{h})$ \\
\hline$k_{p 1}$ & 32 & $\mathrm{FAU} /(\mathrm{g}$ active DW $\mathrm{h})$ \\
\hline$k_{p 2}$ & 5000 & $\mathrm{~L} / \mathrm{g}$ \\
\hline$K_{c o r}$ & $10^{-6}$ & $\mathrm{~g} / \mathrm{L}$ \\
\hline$K_{s l}$ & 0.003 & $\mathrm{~g} / \mathrm{L}$ \\
\hline$K_{s 3}$ & 0.006 & $\mathrm{~g} / \mathrm{L}$ \\
\hline$K_{s 4}$ & 0.0006 & $\mathrm{~g} / \mathrm{L}$ \\
\hline$k_{\text {tip.max }}$ & 49 & $\mathrm{~g}$ active DW/(tip h) \\
\hline$n_{s}$ & 0.01 & $\mathrm{~g} \mathrm{glucose} /(\mathrm{g} \mathrm{DW} \mathrm{h})$ \\
\hline$\square$ & 1 & $\mathrm{~g} / \mathrm{cm}{ }^{3}$ \\
\hline$s_{f}$ & 550 & $\mathrm{~g} / \mathrm{L}$ \\
\hline$s_{r e p}$ & 0.0095 & $\mathrm{~g} / \mathrm{L}$ \\
\hline$w$ & 0.67 & $\mathrm{~g} / \mathrm{g} \mathrm{DW}$ \\
\hline$Y_{s p}$ & 5316 & $\mathrm{FAU} / \mathrm{g}$ \\
\hline
\end{tabular}

Table 1. Parameter values 


\begin{tabular}{|l|l|l|}
\hline \multicolumn{1}{|c|}{$\begin{array}{c}\text { State } \\
\text { variable }\end{array}$} & \multicolumn{1}{c|}{$\begin{array}{c}\text { Initial } \\
\text { Value }\end{array}$} & $\begin{array}{c}\text { Measurement } \\
\text { unit }\end{array}$ \\
\hline$x_{e}(0)$ & 0.001 & $\mathrm{~g} / \mathrm{kg}$ \\
\hline$x_{a}(0)$ & 0 & $\mathrm{~g} / \mathrm{kg}$ \\
\hline$x_{h}(0)$ & 0 & $\mathrm{~g} / \mathrm{kg}$ \\
\hline$s(0)$ & 4 & $\mathrm{~g} / \mathrm{L}$ \\
\hline$p(0)$ & 0 & $\mathrm{FAU} / \mathrm{L}$ \\
\hline$V(0)$ & 4 & $\mathrm{~L}$ \\
\hline
\end{tabular}

Table 2. Initial conditions

\subsection{Numerical Optimization}

The optimization of the filamentous fungi fermentation is considered according to the formulation in Problem (2). The optimal trajectories computed numerically using MATLAB are presented in Figure 2. The input parameterization uses five piecewise linear elements. It can be observed that the optimal control sequence contains three arcs (indicated in the feed flow rate curve of Figure 2):

1) Initially, there is a batch phase that corresponds to lowering the substrate concentration.

2) The second arc corresponds to growing the active biomass in order to reduce the batch time and the amount of substrate spent on the maintenance of the inactive biomass.

3) The third phase corresponds to reducing the substrate concentration so as to maximize the instantaneous yield.

This example falls in the scenario studied in Section 3, where instantaneous yield maximization corresponds to extinction. The main part of the solution, i.e. the second and the third phase, corresponds to one singular arc that gives a bell-shaped input profile. In the final phase, extinction is acceptable since the batch is completed before $x_{a}$ reaches zero.

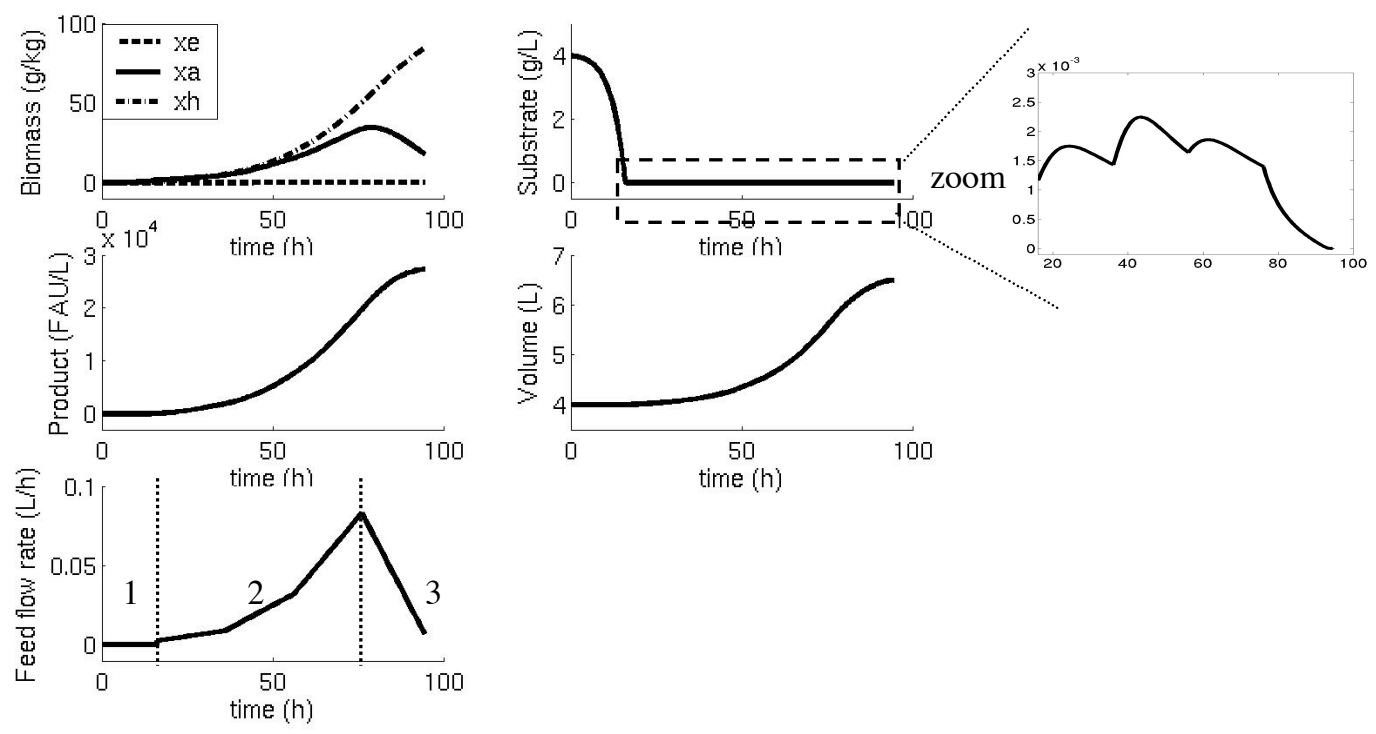

Figure 2. Optimal trajectories obtained via numerical optimization

Additionally, in principle, there is a very short batch phase at the end, where $F=0$ waiting for $d m_{p} / d t=\square$ to be reached. Since the influence of this arc on the cost function is negligible 
compared to the coarseness of the parameterization used, it is not possible to determine it numerically.

It was argued at the end of Section 3 that maintenance of the inactive biomass could be handled by penalizing the final time as in (18), thereby giving rise to a bell shaped profile. This is indeed obtained here for the optimization of filamentous fungi fermentation that include 3 types of biomass.

\section{Conclusion}

This paper has shown that the death of the biomass plays an important role in the yield optimization of bioreactors, especially when the instantaneous yield maximization corresponds to biomass extinction. The analysis is performed on a simplified mathematical model, whereas the ideas are applied to the more detailed model of the filamentous fungi fermentation.

If, instead of final product yield maximization, productivity (amount of product produced/ time elapsed) needs to be maximized, it is unclear whether death would play such a significant role. For such a problem, it is necessary to grow the biomass during the first part of the batch in order to reduce the batch time. Thus, biomass death may not have much of a bearing on the structure of the solution. This represents a future research direction.

\section{Notations}

$a \quad-$ number of tips per unit mass (tips/g extension zone DW)

$\square \quad-$ stoichiometric coefficient for the uptake of substrate

$d \quad-$ hyphal diameter $(\square \mathrm{m})$

$f \quad-$ fraction of the active region (\%)

$F \quad-$ feed flow rate $(\mathrm{L} / \mathrm{h})$

$k_{1} \quad-$ specific branching frequency (tips/(g active DW h))

$k_{2} \quad-$ rate constant $\left(\mathrm{h}^{-1}\right)$

$k_{3} \quad-$ maximal tip extension rate (g active DW/(tip h))

$k_{\text {bran }} \quad-$ specific branching frequency determined by image analysis (tip/( $\left.\square \mathrm{m} \mathrm{h}\right)$ )

$k_{c} \quad-$ constitutive $\square$-amylase production rate (FAU/(g active DW h))

$k_{p l} \quad-$ maximal product formation rate (FAU/(g active DW h))

$k_{p 2} \quad-$ inhibition constant on glucose for $\square$-amylase production $(\mathrm{g} / \mathrm{L})$

$K_{c o r} \quad-$ correction constant for the product formation $(\mathrm{g} / \mathrm{L})$

$K_{s l} \quad$ - saturation constant for branching $(\mathrm{g} / \mathrm{L})$

$K_{s 3} \quad-$ saturation constant for tip extension $(\mathrm{g} / \mathrm{L})$

$K_{s 4} \quad-$ saturation constant for product formation $(\mathrm{g} / \mathrm{L})$

$K_{t i p, \max }$ - maximal tip extension rate determined by image analysis (g active DW/(tip h))

$n_{s} \quad-$ maintenance coefficient (g glucose/(g DW h))

$\square \quad-$ specific growth rate $\left(\mathrm{h}^{-1}\right)$

$p \quad-$ - -amylase concentration $(\mathrm{g} / \mathrm{L})$

$q_{a} \quad$ - growth rate of the active region $(\mathrm{g} /(\mathrm{kg} \mathrm{DW} \mathrm{h}))$

$q_{b} \quad$ - rate of branching $(\mathrm{g} /(\mathrm{kg} \mathrm{DW} \mathrm{h}))$

$q_{d} \quad-$ rate of hyphal cell formation $(\mathrm{g} /(\mathrm{kg} \mathrm{DW} \mathrm{h}))$

$r_{p s} \quad-$ specific $\square$-amylase formation rate (FAU/(g active DW h))

$\square \quad-$ hyphal density $\left(\mathrm{g} / \mathrm{cm}^{3}\right)$

$s \quad-$ substrate concentration $(\mathrm{g} / \mathrm{L})$

$s_{f} \quad-$ concentration of substrate feed $(\mathrm{g} / \mathrm{L})$

$s_{\text {rep }} \quad-$ threshold substrate concentration for inhibition $(\mathrm{g} / \mathrm{L})$

$t \quad-$ time (h)

V $\quad-$ volume (L) 
w $\quad-$ hyphal water content (g/g DW)

$x_{a} \quad-$ concentration of active region $(\mathrm{g} / \mathrm{kg} \mathrm{DW})$

$x_{e} \quad-$ concentration of extension zone $(\mathrm{g} / \mathrm{kg} \mathrm{DW})$

$x_{h} \quad-$ concentration of hyphal region $(\mathrm{g} / \mathrm{kg} \mathrm{DW})$

$Y_{s p} \quad-$ yield coefficient for $\square$-amylase on substrate $(\mathrm{FAU} / \mathrm{g})$

\section{References}

Agger, T., Spohr, A. B., Carlsen, M. and Nielsen, J. (1998). Growth and product formation of aspergillus oryzae during submerged cultivations: verification of a morphologically structured model using fluorescent probes. Biotechnology and Bioengineering 57 (3), 321-329.

Bailey, J. E. and Ollis, D.F. (1986). Biochemical Engineering Fundamentals. MacGraw-Hill. Bryson, A. E. and Ho, Y. C. (1975). Applied Optimal Control. Washington DC: Hemisphere. Lee, J. M. (1992). Biochemical Engineering. Prentice Hall.

Modak, J. M., Lim, H. C. and Tayeb, Y. J. (1986). General characteristics of optimal feed rate profiles for various fed-batch fermentation processes. Biotechnology and Bioengineering 28, 1396-1407.

Srinivasan, B., Palanki, S. and Bonvin, D. (2003). Dynamic optimization of batch processes I. Characterization of the nominal solution. Computers and Chemical Engineering 27, 1-26.

van Impe, J.F., Claes, J. E., Ryckaert, V. G. and Bastin, G. (1994). Characterization of optimal feed rate profiles for fed-batch fermentation processes. Proceedings of the $3 \mathrm{rd}$ IEEE Conference on Control Applications, 1947-1952 